\title{
Neuromuscular Choristoma of the Internal Auditory Meatus
}

\author{
Georgios Nikolaou Christof Röösli Alex Huber Rudolf Probst \\ Department of Otorhinolaryngology, Head and Neck Surgery, University Hospital of Zurich, Zurich, Switzerland
}

\section{Key Words}

Neuromuscular choristoma - Internal auditory meatus • Hamartoma

\begin{abstract}
Choristomas of the internal auditory meatus are exceedingly rare tumors. In most cases, neuromuscular choristomas have initially been misdiagnosed as vestibular schwannomas (VS). No known characteristics in the clinical presentation or in imaging exist distinguishing these tumors from VS, which are the most common tumors at this location [Smith et al.: AJNR Am J Neuroradiol 1997;18:327-329]. We present a case of a neuromuscular choristoma of the 8th cranial nerve that was operated because of growth demonstrated on two MRI scans 3 months apart. We were convinced that this young patient would require treatment sometime in the future, and we believed that an operation at that time had higher chances to preserve the anatomical structures. Histomorphological examination of the tumor revealed a nodular lesion with fascicular and nodular assembled smooth muscle cells, connective tissue and nerve fibers.
\end{abstract}

Copyright $\odot 2012$ S. Karger AG, Basel

\section{Introduction}

The majority (90\%) of tumors in the internal auditory meatus (IAM) are vestibular schwannomas (VS) [1]. Other tumors located in the IAM are facial nerve schwannomas, hemangiomas, meningiomas, lipomas, melanomas, osteomas, metastases and lymphomas [2]. Choristomas of the 8 th cranial nerve are very rare lesions with only 14 reported cases to date [3-6]. Most of them have been misdiagnosed preoperatively as VS [3-6], which is not surprising due to the much higher incidence of VS, the similar clinical presentation of the two tumors, and because a preoperative biopsy of a lesion in the IAM is not feasible.

The exact definition of the term choristoma causes some confusion. Chorista is the malformation of a tissue resulting from the spread of embryonic tissue into aberrant locations, and choristoma is the tumor which results from chorista or autonomous, continuous development of this tissue in aberrant areas. Choristomas are not tumors consisting of a specific cell type; they can potentially form a developed organ. Essentially, choristomas are masses of normal tissue in aberrant locations. They may contain smooth muscle fibers, fibrous tissues and nerve fibers. They are sometimes diagnosed as hamartomas, but while hamartomas are also non-neoplastic proliferations of histologically normal tissues, they are not aberrant and occur at sites where those tissues are normally found [7-10].

Dr. Georgios Nikolaou

Department of Otorhinolaryngology, Head and Neck Surgery

University Hospital of Zurich

CH-8091 Zurich (Switzerland)

E-Mail georgios.nikolaou@usz.ch 


\section{Case Report}

A 29-year-old woman presented with a right-sided hearing loss, unspecific vertigo complaints and an intermittent tinnitus. Otoscopy and vestibular examinations were normal. Audiometric studies revealed a mild to moderate sensorineural hearing loss of $25-35 \mathrm{~dB}$ in the frequency range of $0.5-4 \mathrm{kHz}$ (fig. 1). Speech discrimination in the Freiburger monosyllabic word test was 85\% at $100 \mathrm{~dB}$ SPL (fig. 2). An MRI revealed a $6 \times 3 \mathrm{~mm}$ lesion in the IAM (fig. 3). The lesion was enhanced strongly after the injection of gadolinium, which was considered highly suspicious for VS. A follow-up MRI 3 months after the initial diagnosis of the tumor showed a tumor size of $9 \times 4 \times 5 \mathrm{~mm}$. Surgery was proposed to the patient at this point because of the growth and the high probability of the necessity of treatment in the future. Chances to preserve function were judged to be best with early surgery.

The tumor was removed by a retrosigmoidal approach. After opening the IAM, a rubbery, hard tumor was found attached primarily to the inferior and superior vestibular nerves (fig. 4). A complete removal of the tumor could be achieved only after sectioning the vestibular and cochlear nerves because both nerves and the tumor were tangled. The histopathological study revealed a benign nodular lesion that consisted of smooth muscle cells in a fascicular and nodular arrangement, connective tissue and nerve fibers. The histopathological diagnosis was a neuromuscular choristoma of the 8th cranial nerve (fig. 5, 6).

After surgery the patient demonstrated a right-sided cochleovestibular loss due to the section of the vestibular and cochlear nerve, without facial nerve palsy. She made a rapid recovery from the postoperative dizziness supported by physiotherapy.

One week after discharge the patient presented to the emergency room with a progressive headache not responding to analgesics. The CT scan revealed a thrombosis of the vena jugularis interna and the right-sided sinus sigmoideus. The patient received oral anticoagulation therapy for 3 months. Despite the oral anticoagulation and the non-progressive thrombosis of the vena jugularis interna and the right-sided sinus sigmoideus, the disabling headache persisted for several months. The neurological examination was normal, with the exception of a right-sided hearing

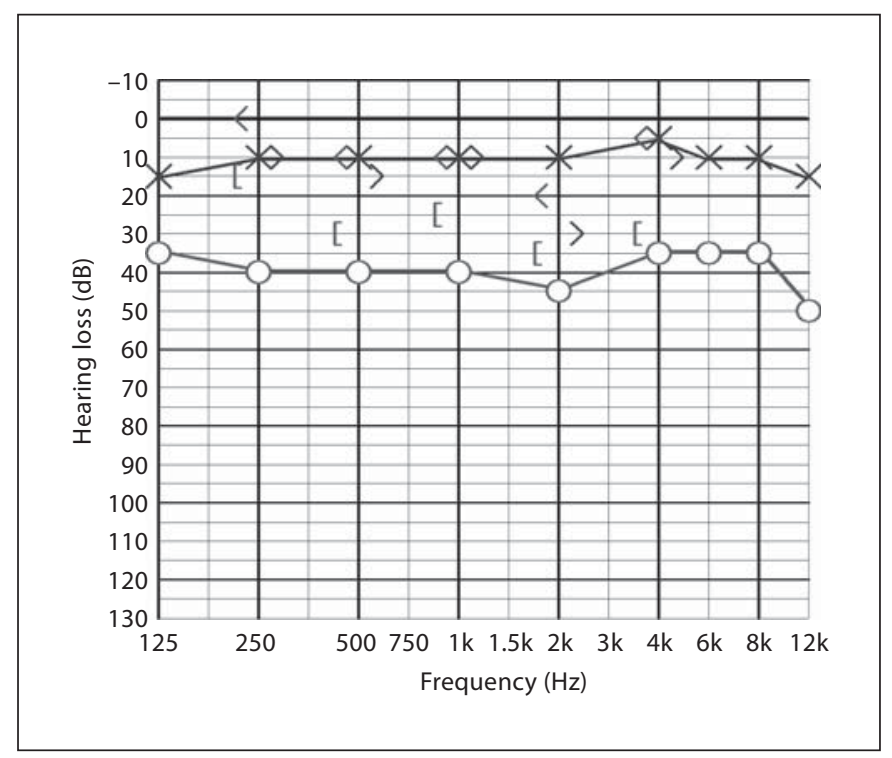

Fig. 1. Audiometric studies revealed a mild to moderate sensorineural hearing loss of $25-35 \mathrm{~dB}$ in the frequency range of $0.5-4$ $\mathrm{kHz}$.

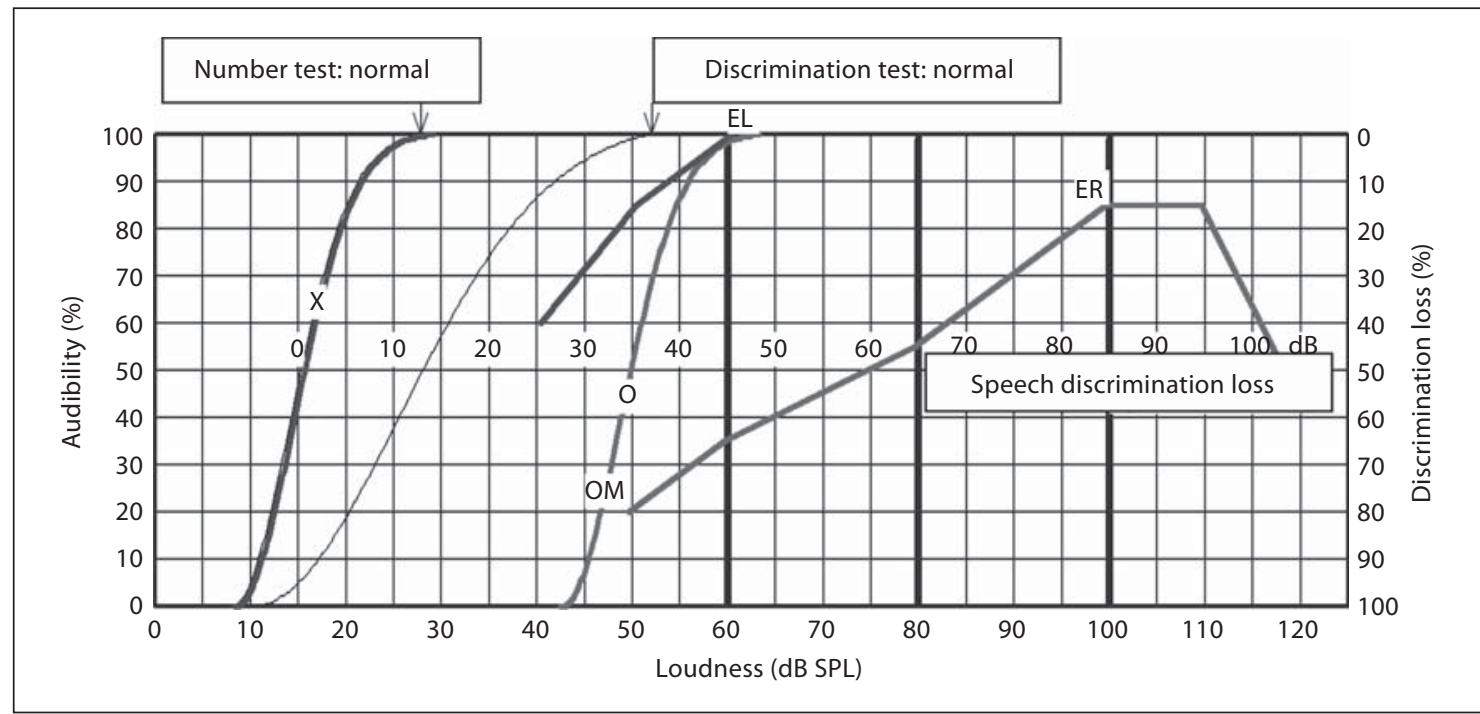

Fig. 2. Speech discrimination in the Freiburger monosyllabic word test was $85 \%$ at $100 \mathrm{~dB}$ SPL. EL = air conduction left ear (Freiburger monosyllabic word test); ER = air conduction right ear (Freiburger monosyllabic word test); $\mathrm{O}=$ air conduction right ear (bisyllabic word test); $\mathrm{OM}=$ air conduction right ear with masking (bisyllabic word test). 


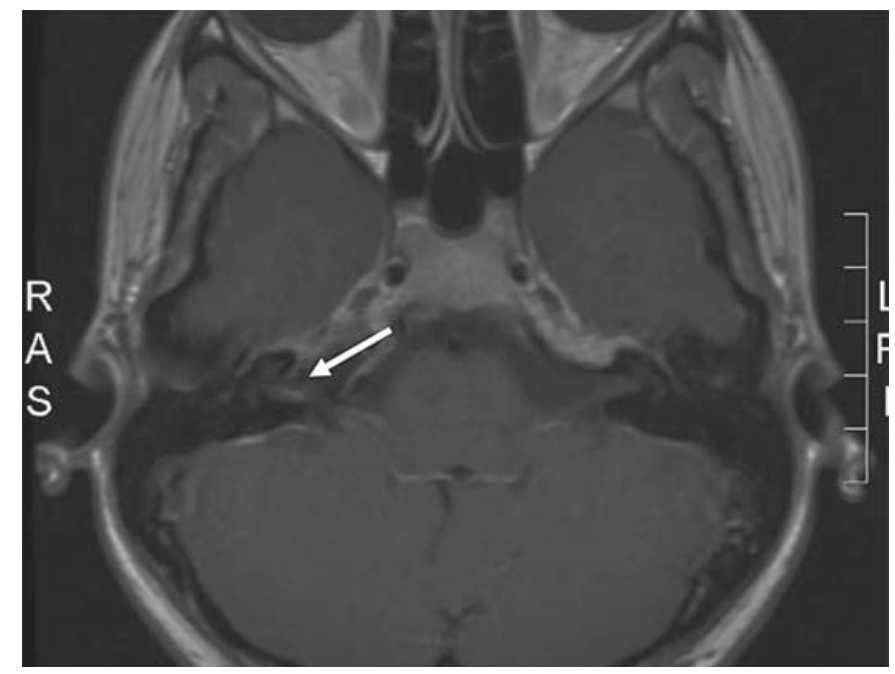

Fig. 3. MRI of a $6 \times 3 \mathrm{~mm}$ lesion in the IAM. Arrow showing the gadolinium enhancing tumor.

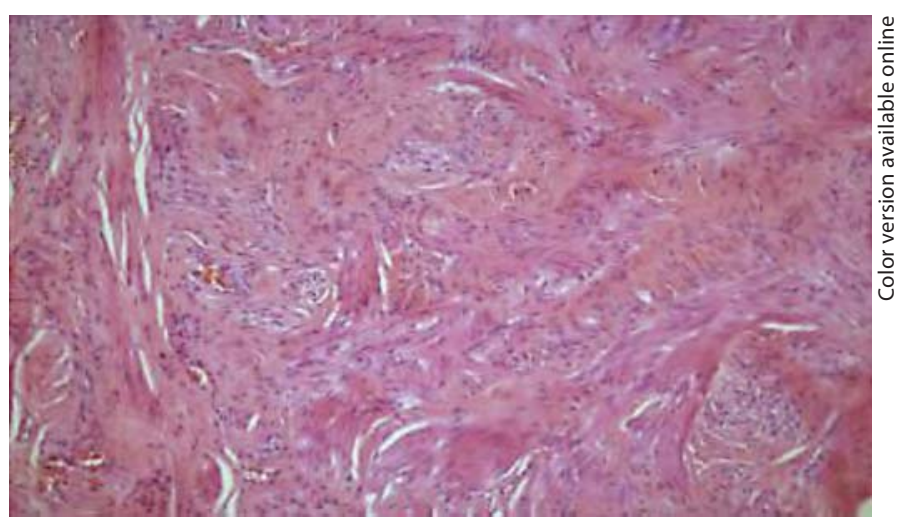

Fig. 5. Spindle cells (Schwann cells) that correspond to nerve fibers and muscle cells in fascicular arrangement (magnification $\times 20$, hematoxylin and eosin stain).

loss, and another MRI scan showed no specific findings. A headache due to medication overuse was diagnosed and was treated by stopping the overused medication and setting up a preventive regimen with carbamazepine.

\section{Discussion}

Neuromuscular choristomas in the IAM are exceedingly rare lesions. The tumor in our report consisted of smooth muscle tissue, nerve fibers and adipose tissue. Smooth muscle tissue does not represent a component of

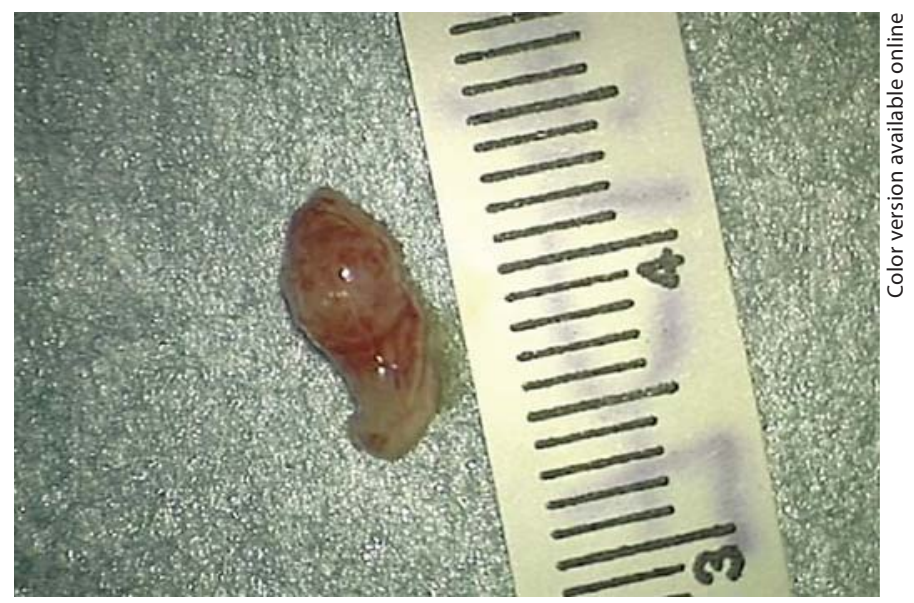

Fig. 4. A rubbery, very hard tumor was removed from the inferior and superior vestibular nerve.

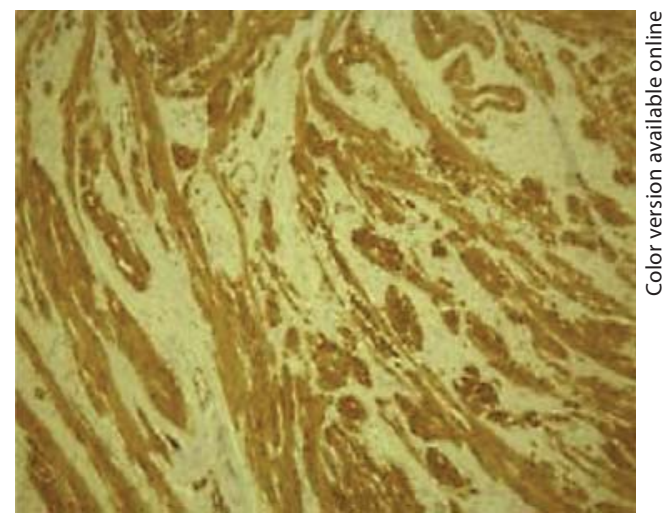

Fig. 6. Smooth muscle antigen for smooth muscle (magnification $\times 20)$.

cranial nerves and we suggest that the correct classification for this lesion is neuromuscular choristoma and not hamartoma. We found only 14 reports of cases of choristomas of the 8th cranial nerve, which were all removed surgically. The reports did not say whether the choristomas were removed due to growth or due to the symptoms they caused. All contained smooth muscle cells as a major component, connective tissue and nerve fibers. In 4 of 11 cases published in a complete clinical review, the tumors contained also skeletal muscle cells which were not found in our case [6]. All patients suffered from a hearing loss with the exception of 1 patient with hyperacusis and 2 
additional patients in whom the hearing status was not reported. Two of the patients with hearing loss presented additionally with tinnitus, 1 patient reported additionally the loss of balance and another patient tinnitus and the loss of balance [3-6].

Little is known about the nature and history of these tumors. A wide range of behavior of these tumors in other locations is described in the literature. No growth at all or very slow growth is the typical behavior. Comments on the progression as well as spontaneous remission after incomplete removal can be found, but the dynamic behavior of choristomas in the IAM has not been reported $[3-5,8]$. The only way to monitor the growth of tumors in the IAM is serial MRI scans. If a tumor is growing, treatment is generally recommended. The tumor in our case seemed to grow slowly when comparing between the first and the follow-up MRI scan 3 months later. It might be beneficial to document the size of a tumor in the IAM by calculating its volume. Assuming the tumor is an ellipsoid as an approximation, the volume was initially 527 $\mathrm{mm}^{3}$, while it increased to $754 \mathrm{~mm}^{3}$ within 3 months.

How can choristomas be differentiated from other tumors in the IAM? Contrast-enhanced MRI may be helpful. Tumors in the IAM can be classified into 2 groups depending on their location: there are extra-axial lesions (outside the brain parenchyma) such as VS, meningiomas, epidermoid cysts and paragangliomas, and rare tumors such as schwannomas of the 5th, 6th, 7th, 9th, 10th, 11 th or 12th cranial nerve, and vascular lesions; and there are intra-axial lesions (within the brain parenchyma). The latter are less common in MRI at this location and include astrocytomas, ependymomas, papillomas, he- mangioblastomas and metastases $[11,12]$. Schwannomas of the facial nerve may extend into the labyrinthine facial nerve canal, and this feature could distinguish them from other tumors. Meningiomas can be distinguished from other tumors by the presence of calcification, dural tail and bone infiltration [2]. Vascular tumors like hemangiomas tend to be more hyperintense in MRI. Lipomas can usually be recognized by their hyperintense signal in $\mathrm{T}_{1}$-weighted MRI and can be confirmed by fat suppression techniques. Osteomas are hypointense in all sequences and non-enhancing after contrast [11]. However, choristomas cannot be distinguished from other tumors in the IAM such as VS [1]. Therefore, choristomas are usually misdiagnosed as the more common VS initially. Due to a lack of access for biopsy, lesions in the IAM are often only diagnosed after surgical removal. It remains unclear whether a non-surgical therapy such as radiation therapy is an alternative treatment option for growing choristomas.

\section{Conclusion}

In summary, a neuromuscular choristoma is an exceedingly rare tumor in the IAM. The preoperative distinction from schwannomas does not seem possible. The definitive diagnosis can be made only by histopathological examination after surgical removal. The natural behavior of this tumor is not known, but observing an expansion of the lesion documented in serial MRI is possible, and growing tumors should be treated.

\section{References}

1 Babin RW, Fratkin JD, Cancilla PA: Hamartomas of the cerebellopontine angle and internal auditory canal: report of two cases. Arch Otolaryngol 1980;106:500-502.

$\checkmark 2$ Sriskandan N, Connor SE: The role of radiology in the diagnosis and management of vestibular schwannoma. Clin Radiol 2011;66: 357-365.

3 Smith MM, Thompson JE, Thomas D, et al: Choristomas of the seventh and eighth cranial nerves. AJNR Am J Neuroradiol 1997; 18:327-329.

4 Ajal M, Roche J, Turner J, Fagan P: Unusual lesions of the internal auditory canal. J Laryngol Otol 1998;112:650-653.

Neuromuscular Choristoma of the

ORL 2012;74:246-249
9 Gersdorff MC, Decat M, Duprez T: Neuromuscular hamartoma of the internal auditory canal. Eur Arch Otorhinolaryngol 1996; 253:440-442. Smooth muscle choristoma of the internal auditory meatus. Eur Arch Otorhinolaryngol 2005;262:834-838.

6 Wu SS, Lo WW, Tschirhart DL, Slattery WH 3rd, Carberry JN, Brackmann DE: Lipochoristomas (lipomatous tumors) of the acoustic nerve. Arch Pathol Lab Med 2003;127:14751479.

7 Apostolides PJ, Spetzler RF, Johnson PC: Ectomesenchymal hamartoma (benign 'ectomesenchymoma') of the VIIIth nerve: case report. Neurosurgery 1995;37:1204-1207.

8 Carvalho GA, Matthies C, Osorio E, Samii M: Hamartomas of the internal auditory canal: report of two cases. Neurosurgery 2003; 52:944-948, discussion 948-949.
0 Goda M, Isono M, Karashima A, Kasai N, Kobayashi H: Hamartoma in the internal auditory canal. J Clin Neurosci 2003;10:111113.

11 Bigelow DC, Eisen MD, Smith PG et al: Lipomas of the internal auditory canal and cerebellopontine angle. Laryngoscope 1998;108: 1459-1469.

12 Maya MM, Lo WWM, Kovanlikaya I: Temporal bone tumors and cerebellopontine angle lesions; in Som PM, Curtin HD (eds): Head and Neck Imaging, 2 vol. St. Louis, Mosby, 2003, pp 1275-1352. 